\title{
Relativity, Dimensionality, and Existence
}

\author{
Vesselin Petkov \\ Science College, Concordia University \\ 1455 de Maisonneuve Boulevard West \\ Montreal, Quebec, Canada H3G 1M8 \\ vpetkov@alcor.concordia.ca
}

Since the [relativity] postulate comes to mean that only the four-dimensional world in space and time is given by phenomena, but that the projection in space and in time may still be undertaken with a certain degree of freedom, I prefer to call it the postulate of the absolute world.

H. Minkowski [1, p. 83]

\begin{abstract}
The basic idea is to present the essentials of relativity from the Minkowskian point of view, that is, in terms of the geometry of space-time... because it is to me (and I think to many others) the key which unlocks many mysteries. My ambition has been to make space-time a real workshop for physicists, and not a museum visited occasionally with a feeling of awe.
\end{abstract}

J. L. Synge $[2$, p. vii $]$

\section{Introduction}

A hundred years have passed since the advent of special relativity and 2008 will mark another important to all relativists anniversary - one hundred years since Minkowski gave his talk "Space and Time" on September 21, 1908 in which he proposed the unification of space and time into an inseparable entity - spacetime. Although special relativity has been an enormously successful physical theory no progress has been made in clarifying the question of existence of the objects represented by two of its basic concepts - spacetime and worldlines (or worldtubes in the case of extended bodies). The major reason for this failure appears to be the physicists' tradition to call such questions 
of existence philosophical. This tradition, however, is not quite consistent. In Newtonian mechanics physicists believe that they describe real objects whenever they talk about particles - one of the basic concepts of Newtonian physics. The situation is the same in quantum physics - no one questions the existence of electrons, protons, etc. Then why should the question of existence of worldtubes (representing particles in relativity) be regarded as a philosophical question?

The most probable answer a physicist would give is that the concepts of spacetime and worldtubes belong to the four-dimensional representation of special relativity, whereas in its three-dimensional formulation these concepts are not used. Since both representations of special relativity are equivalent it appears that one should not worry about the existence of spacetime and worldtubes. Most physicists and especially relativists appear to believe that by emphasizing the equivalence of the three- and four-dimensional descriptions of the world provided by special relativity the issue of the existence of spacetime has been shown to be a non-issue. However, as we will see that would be quite a premature attempt to close such a fundamental issue.

The three- and four-dimensional representations of special relativity are equivalent, but I think it is a valid question to ask which of them is the adequate description of the world. One is naturally tempted to immediately question the validity of such a question by pointing out that these are just two descriptions and to ask which is the right one is meaningless. For instance, one could explain that such a question is as meaningless as to ask whether the Newtonian or the Lagrangian formulation of classical mechanics is more adequate. Once again this situation clearly demonstrates that each case in science (and not only in science) should be dealt with separately. The equivalence of the two representations of special relativity are drastically different from the equivalence of the Newtonian and Lagrangian formulations of classical mechanics. The three-dimensional formulation of special relativity represents reality as a three-dimensional world which evolves in time, whereas according to the four-dimensional formulation the world is four-dimensional with time entirely given as the forth dimension. As the world is either three-dimensional or four-dimensional, it is clear that either the three-dimensional or the fourdimensional description of the world is the correct one in a sense that only one of them correctly reflects the dimensionality of the world ${ }^{1}$. Also, physical bodies are either three-dimensional or four-dimensional objects. Therefore, not only is the question of the dimensionality of the world and the physical objects not a non-issue, but it is one of the most fundamental issues of the 21st century physics. Moreover, it is natural to address the question of the dimensionality of the world on the macroscopic scale according to relativity

\footnotetext{
${ }^{1}$ Both the three- and four-dimensional descriptions of the world are correct in terms of their adequate descriptions of the physical phenomena, but in terms of the dimensionality of the world they cannot be both correct since the world is not both three- and four-dimensional.
} 
first, before dealing with the reality of extra dimensions introduced by more recent physical theories.

The main purpose of this paper is to demonstrate that the analysis of the kinematical effects of special relativity holds the key to answering the question of the dimensionality of the world. It is shown that these effects and the experiments which confirmed them would be impossible if the world were threedimensional. Section 2 shows that relativity of simultaneity, conventionality of simultaneity, and the existence of accelerated observers in special relativity would be impossible if the world were three-dimensional. Section 3 deals with the dimensionality of physical objects and demonstrates that the relativistic length contraction and the twin paradox would be impossible if the physical bodies involved in these relativistic effects were three-dimensional objects.

\section{Special relativity is impossible in a three-dimensional world}

Perhaps most physicists would disagree with the statement in the section title. They would probably point out the well-known fact that special relativity was initially formulated in a three-dimensional language. However, the only thing this fact says is that the relativistic effects can be given a three-dimensional description ${ }^{2}$; it says nothing about whether or not these effects would be possible in a three-dimensional world.

The best way to prove the statement in the section title is to analyze the kinematical effects of special relativity in order to reveal their true physical meaning. Such an analysis is especially needed since all physics books on relativity merely describe the relativistic effects without addressing the questions of the dimensionality and existence of the physical objects involved in these effects. Once those questions have been explicitly dealt with it becomes evident, as we will see below, that the physical meaning of the kinematical relativistic effects is profound - these effects turn out to be manifestations of the four-dimensionality of the physical world. That is why they would be impossible in a three-dimensional world.

\subsection{Relativity of simultaneity is impossible in a three-dimensional world}

Let us start the analysis of relativity of simultaneity with the question "What is the dimensionality of the world according to relativity?" As the observable world is three-dimensional it is understandable why according to the

${ }^{2}$ Obviously, a description itself does not tell anything about the dimensions of what is described. For instance, the $x-y$ plane can be described either in twodimensional or one-dimensional language (regarding the $y$ dimension as a parameter). 
widely accepted view, called presentism, reality is also a three-dimensional world which exists at the constantly changing present moment. But the dimensionality of the observable world tells us nothing about the dimensionality of the world itself since the observable world is not what is real according to the presentist view. It consists only of past events ${ }^{3}$ - the past light cone. Therefore, the observable three-dimensional world does not constitute even a three-dimensional world since such a world consists of all events that are simultaneous at a given moment, whereas the observable world is a set of events belonging to different moments of time (the more distant an event is the more in the past it belongs).

If we assume that reality itself (not only the observable part of it) is also a three-dimensional world, it can be easily shown that relativity of simultaneity is impossible. As a three-dimensional world is defined as everything that exists simultaneously at the present moment, it follows that the three dimensional world (as the only existing entity) will be common to all observers ${ }^{4}$ in relative

\footnotetext{
3 "The key-word in relativity is event" [3, p. 105]. "An event marks a location in spacetime" [4, p. 10]. In most relativity books 'event' is used as a synonym of 'worldpoint' or 'spacetime point' $[2$, pp. 5-6], [5, p. 6], [6, p. 2-1, p. GL-4], $[7$, p. 427], [8, p. 53], [9, p. 4], [10], [11, pp. 1, 9], [12, p. 25], [13], [14, p. 23], $[15],[16$, p. 66]. This appears to be quite natural since an event, defined as a worldpoint, is determined only by its space and time coordinates and is not associated only with physical bodies or phenomena. The definition of a concept is of course a matter of convention and, for example, one can define 'event' to mean 'a worldpoint at which a physical object is located'. However, such a definition invites a number of misconceptions. For instance, one is tempted to talk about different events that happen with the same body. But since a body is implicitly regarded as a three-dimensional object it would mean that different events happen with the same three-dimensional object, which makes no sense in relativity where a physical body is represented by a four-dimensional worldtube. A worldtube, or simpler, a worldline consists of different worldpoints, which represent different three-dimensional objects. Therefore, to talk about different events happening with the same three-dimensional body is clearly wrong in the framework of relativity (but it is fine in the pre-relativistic physics). The same physical body in relativity means the same four-dimensional object - the worldtube of the body. The different events which constitute the worldtube involve different threedimensional objects - the physical body at different moments of its proper time. In this paper I will use the widely accepted definition of event as a worldpoint, but will make that definition more explicit - an event is defined as a three-dimensional object, a field point, or a space point at a given moment of time [17, p. 56]. I would like to stress specifically that all events of spacetime (like all points of any dimensional space) have the same status of existence; otherwise, if the events of just one three-dimensional hypersurface were existent, one could not talk about spacetime at all.

4 "The word 'observer' is a shorthand way of speaking about the whole collection of recording clocks associated with one free-float frame. No one real observer could easily do what we ask of the 'ideal observer' in our analysis of relativity. So it is best to think of the observer as a person who goes around
} 
motion; therefore they will have a common set of simultaneous events, which means that relativity of simultaneity is indeed impossible. The conclusion that relativity of simultaneity is possible only in a four-dimensional world seems unavoidable provided that existence is regarded as absolute (frame- or observerindependent) - the observers in relative motion can have different sets of simultaneous events only in a four- (or higher-) dimensional world; these sets constitute different three-dimensional 'cross-sections' of such a world ${ }^{5}$. In this sense relativity of simultaneity is a manifestation of the four-dimensionality of the world.

The conclusion that relativity of simultaneity implies a four-dimensional world looked unavoidable to Minkowski [1], Rietdijk [24], Putnam [25], Maxwell $[26]$, and to all who agree with the above argument ${ }^{6}$. However, one can formulate three objections to the relativity of simultaneity argument.

The first objection was raised by Stein [30, 31]. He pointed out that the relativity of simultaneity argument was flawed since it employed the prerelativistic concept of distant present events, whereas according to the relativistic division of events into past, present, and future one can attribute presentness only to a single event (Fig. 1). The relativity of simultaneity argument is clearly based on the pre-relativistic view on reality that only the present, defined as the three-dimensional world at the present moment, is real. However, the present is the set of distant present events - everything that exists simultaneously at the moment 'now'. Therefore the argument that due to relativity of simultaneity two observers in relative motion have different presents, which is possible only in a four-dimensional world, does employ the concept of distant present events.

reading out the memories of all recording clocks under his control. This is the sophisticated sense in which we hereafter use the phrase 'the observer measures such-and such'." [4, p. 39] (see also [6, p. 1-18]). The concept 'observer' (or 'experimenter' $[7$, p. 428]) is widely used in relativity [8, p. 95], [12, p. 48], [14, p. $23],[16$, p. 67] [18] in many cases as a synonym of 'reference frame' [11, p. 2], [20]-[23]. If during an experiment the observer is located at the origin of an inertial reference frame, which has been constructed in the standard way (see for instance $[9$, p. 6] $)$, then there is no difference between 'observer' and 'reference frame'.

${ }^{5}$ As sometimes physicists and philosophers are inclined to think that the threedimensionalist view can somehow be made consistent with the relativistic picture of the world they should obviously explain how relativity of simultaneity is possible in a three-dimensional world, which by definition contains just one set of simultaneous events, whereas two observers in relative motion have different sets of simultaneous events.

${ }^{6}$ Einstein himself [27], Weyl [28], Weingard [29], and a number of physicists and philosophers of science view relativity as clearly supporting the fourdimensionalist view. In 1952 Einstein wrote: "It appears... more natural to think of physical reality as a four-dimensional existence, instead of, as hitherto, the evolution of a three-dimensional existence" [27]. 


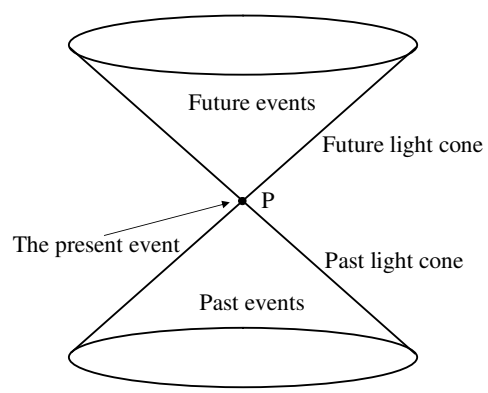

Fig. 1. In relativity the division of events into past and future is with respect to one event, which is considered present.

Due to the fact that the three-dimensional world is the set of all distant present events Stein's objection is in fact directed against the view that reality is a three-dimensional world [17, p. 123], [32]. Thus, by demonstrating that the concept of distant present events (and therefore the view that the world is three-dimensional) contradicts special relativity, Stein de facto sharply raised the question of the dimensionality of the world according to relativity but did not address it.

The same objection is also raised by Ohanian [33]: "[I]n relativistic physics there is no absolute time, and no physical meaning can be attached to simultaneity at different locations." That is precisely the case, since "in special relativity, the causal structure of space-time defines a notion of a 'light cone' of an event, but does not define a notion of simultaneity" [34]. But the crucial question is "What is the physical meaning of the relativistic fact that "no physical meaning can be attached to simultaneity at different locations'?" As we have seen the immediate implication is obvious - the world cannot be threedimensional since it is defined as everything (at different locations) that exists simultaneously at a given moment. If reality were a three-dimensional world, then a clear physical meaning had to be attached to simultaneity at different locations - a class of events (at different locations) would be simultaneous because it is only these events that are real at the present moment. Ohanian did not explicitly rule out the three-dimensionality of the world. Instead he took a careful position: "The physical world inhabits this 4-D spacetime, but the physical world does not occupy all of spacetime - it occupies only a subspace of spacetime. The dimension of the physical world is the dimension of this occupied subspace. It is obvious that this occupied subspace is at least 3-D." It turns out, however, that this view is inconsistent with relativity for the following two reasons:

- If the physical world is represented by a three-dimensional subspace of spacetime, the contradiction with relativity, as we have seen, is inescapable - the set of simultaneous events, which constitute the three-dimensional world, would be common to all observers in relative motion and there- 
fore no relativity of simultaneity would be possible. Even if the threedimensional physical world is regarded as somehow 'more existent' than the remaining part of spacetime, relativity of simultaneity would again be impossible - what is 'more existent' for one observer must be also 'more existent' for all other observers in relative motion with respect to the first one which means that simultaneity would be absolute. An assumption that every observer has his own set of 'more existent' simultaneous events amounts to a relativization of existence which, as we will see below, contradicts relativistic facts which are not based on relativity of simultaneity.

- If the physical world occupies not all of spacetime, but a four-dimensional subspace of it - the acausal spacetime region outside of the light cone at an event (Fig. 1) - it immediately follows that the events in the past and future light cone are as real as the events lying outside of the light cone [29]. To see this assume that the physical world is represented by all events lying outside of the light cone at event $P$ (Fig. 2), i.e. all events that are not causally related to $P$. On this view the events in the past and the future light cone are not real. But if we consider a second light cone at event $P^{\prime}$, most of the past and future events associated with the first light cone lie, as seen in Fig. 2, in the spacetime region outside of the second light cone and are therefore real. As for any event $P$ the division of events into past and future is invariant (since it is an intrinsic, absolute feature of spacetime), it follows that what is real for an observer at $P^{\prime}$ should be real for an observer at $P$ as well. Therefore if one starts with the assumption that the physical world is the spacetime region outside of the light cone at an event, it does follow that the physical world is represented by all events of spacetime.

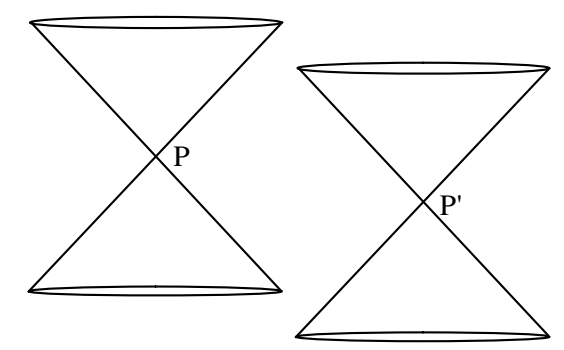

Fig. 2. If one assumes that only the events lying outside of the light cone at $P$ are real, by the same argument the events lying in the area outside of the light cone at $P^{\prime}$ (which contains most of the events of the past and future events of the light cone at $P$ ) are also real and therefore all events of spacetime are real.

The second objection against the relativity of simultaneity argument is based on the conventionality of simultaneity, which itself follows from the (invariant) relativistic division of events into past, present, and future (Fig. 
1). Weingard [29] was the first to raise this objection ${ }^{7}$ against the relativity of simultaneity argument used by Putnam [25]. Ohanian's formulation of this objection is [33]:

Any of the hypersurfaces in the 4-D acausal region between the absolute past and future regions can be regarded as an equal-time hypersurface for the point $P$, provided that the surface includes the point $\mathrm{P}$ and that every point on the surface is in the acausal region of every other point on the surface (that is, the surface is "spacelike"). Thus, in relativistic physics there is no unique, physically distinguished definition of simultaneity. To decide what is simultaneous, we need to adopt some prescription for the construction of equal-time hypersurfaces, or, equivalently, a procedure for clock synchronization. Simultaneity then becomes a matter of convention, without any direct physical significance.

As seen from the quote this objection is an elaboration of the first one since it emphasizes that no three-dimensional hypersurface in the spacetime region outside of the light cone at a given event is in any way physically distinguishable from the other hypersurfaces in the same spacetime region. This means that no hypersurface is privileged on account of its being associated with a single three-dimensional world, which is a set of distant present events; if reality were a three-dimensional world, then the hypersurface associated with it would be naturally privileged. That is what relativity tells us, but again the crucial question is "What is the physical meaning of the relativistic fact that 'simultaneity becomes a matter of convention, without any direct physical significance'?"

Like in the case of the first objection an immediate implication from that relativistic fact is that the physical world cannot be three-dimensional. If the world were three-dimensional it would be represented by one hypersurface (a set of simultaneous events) that is physically distinguishable ${ }^{8}$ from the other hypersurfaces in the spacetime region outside of the light cone at event $P$, which is not the case. A second argument is the following - since one is free to choose which hypersurface to regard as a set of simultaneous events at $P$, which means that simultaneity is a matter of convention, it follows that what exists would be also a matter of convention if reality were a three-dimensional world (defined as the set of simultaneous events at a given moment) [29], [35]. Although this argument against the three-dimensionality of the world looks quite convincing (since what exists does not depend on our free choice) it seems not everyone agrees with it. For instance, Ohanian [33] offers the

\footnotetext{
${ }^{7}$ Despite criticizing Putnam for his conclusion that relativity of simultaneity implies a four-dimensional world Weingard arrived at the same conclusion on the basis of conventionality of simultaneity.

${ }^{8}$ It would be physically distinguishable merely because there would be just one hypersurface that represents the single three-dimensional world that exists.
} 
following counter-argument: "[I]f the 3-D world is unobservable, I am content to fix it by convention." If what exists is a three-dimensional world it is indeed unobservable at the moment it exists, but does this mean that its existence depends in any way on our choice?

Although viewing reality as a three-dimensional world directly contradicts relativity (since such a view implies that a hypersurface in the spacetime region lying outside of the light cone at an event is physically distinguished), let us assume, for the sake of the argument, that reality is indeed a single three-dimensional world represented by a hypersurface that is orthogonal to the worldtube of an inertial observer. This means that the Einsteinian synchronization of distant clocks has been used (i.e. the back and forth velocities of light have been taken to be equal). In this case we will have the ordinary relativistic effects (relativity of simultaneity, length contraction, time dilation, etc.). The experiments designed to test these effects have confirmed them. Let us now choose a non-Einsteinian synchronization of distant clocks, which would mean that the hypersurface representing the three-dimensional world would not be orthogonal to the worldtube of the inertial observer and the back and forth velocities of light would not be equal. All relativistic effects would be again derived, but the expressions for length contraction and time dilation, for instance, would be more complicated [36]. If we decide to perform an experiment to test length contraction, we should expect that it would confirm either the expression derived on the basis of the Einsteinian synchronization of distant clocks or the expression employing the non-Einsteinian synchronization. The reason for this expectation is clear - the hypersurface that represents the existing three-dimensional world would either intersect the worldtube of the measured meter stick at a given angle ('cutting off' a three-dimensional meter stick of a given length) or at a different angle ('cutting off' another three-dimensional meter stick of a different length). Thus, the fundamental belief that what exists does not depend on our choice has observational consequences if the world is three-dimensional. It should be stressed that if reality were a three-dimensional world the experiment would confirm only one of the expressions for length contraction.

However, if we perform that experiment it would confirm both expressions for length contraction. Does this mean that it is we who decide whether or not the hypersurface representing a three-dimensional world would be orthogonal to the worldtube of the inertial observer? Yes, it does, but not because we can fix the three-dimensional world by convention. The reason is that the physical meaning of conventionality of simultaneity also turns out to be profound. Simultaneity can be a matter of convention 'without any direct physical significance' only if the spacetime region outside of a light cone at an event exists as a whole. In this case conventionality of simultaneity is trivial (with no physical significance) simply because the whole four-dimensional region exists and we are free to choose any hypersurface in it (because we have from where to choose!). But as we have seen, the existence of the spacetime region outside of the light cone implies the existence of the whole spacetime. Thus, convention- 
ality of simultaneity is possible only if spacetime is a real four-dimensional entity. If reality were a three-dimensional world, simultaneity would be absolute and no conventionality would be possible - in that case there would exist just one three-dimensional world (a single set of simultaneous events) and one would not have from where to choose his three-dimensional world.

The third objection against the relativity of simultaneity argument is more philosophical in nature. It calls for the relativization of existence. As mentioned above, the relativity of simultaneity argument implicitly regards existence as absolute - having different sets of simultaneous events two observers in relative motion have different three-dimensional worlds which means that both three-dimensional worlds must exists for every observer (existence is absolute!). But this is only possible in a four-dimensional world. The objection is: "Why should existence be absolute? Relativity relativized motion, simultaneity, and now it is the turn of existence to be relativized. If this is done, each of the observers in relative motion would claim that only his three-dimensional world would exist and would deny the existence of the three-dimensional worlds of the other observers. This relativized version of the three-dimensionalist view preserves the three-dimensionality of the world but is undefendable for the following reasons:

- It is in obvious contradiction with relativity since the three-dimensional world of an observer would be represented by a hypersurface (a set of distant present event) lying outside of the light cone, which would be physically distinguished from the other hypersurfaces in the same spacetime region.

- The relativization of existence is based on relativity of simultaneity and has no justification in relativistic situations where no relativity of simultaneity is involved. Moreover, as we will see below, the relativized version of the three-dimensionalist view, which regards the world and the physical objects as three-dimensional, is in direct contradiction with conventionality of simultaneity, the existence of accelerated observers in special relativity, and the twin paradox.

\subsection{Conventionality of simultaneity is impossible in a three-dimensional world}

We have seen that conventionality of simultaneity is a consequence of the relativistic division of events into past, present, and future (Fig. 1) - any observer at event $P$ is free to choose which hypersurface lying outside of the light cone at $P$ can be regarded by him as the set of events that are simultaneous for him at $P$. As discussed above the profound physical meaning of this freedom is that the whole four-dimensional spacetime region outside of the light cone at $P$ must exist. That is why - because it exists - we can choose different hypersurfaces from it. Otherwise, if reality were a three-dimensional world no such freedom in choosing different hypersurfaces, representing different threedimensional worlds, would be possible. 
One may be left with the impression that it is the four-dimensional representation of special relativity and particularly the concept of a light cone, which demonstrated that conventionality of simultaneity is impossible in a three-dimensional world. Moreover, it is generally believed that the fourdimensional picture of the world itself became possible only after Minkowski's talk on space and time [1] in 1908. In fact, that picture is logically contained in the original formulation of special relativity by Einstein in 1905. Minkowski was the first to realize that the relativistic effects are manifestations of a world of higher dimensions - a four-dimensional world with time being the extra dimension. That is why he pointed out that the essence of special relativity is not relativity of space, time, and other physical quantities as the principle of relativity had been interpreted to mean in the early years of special relativity. According to Minkowski that principle should be replaced by 'the postulate of the absolute world' $[1$, p. 83] since a rigorous analysis of the physical meaning of the relativity principle reveals that reality is an absolute (frame-independent) four-dimensional world.

And indeed if we explicitly address the question of the physical meaning of the relativistic effects (assuming that we do it, say, in 1906) it does become evident that these effects are manifestations of the four-dimensionality of world. We have seen this in the case of relativity of simultaneity - once the key role of simultaneity in the definition of a three-dimensional world has been taken into account the conclusion that relativity of simultaneity is impossible in a three-dimensional world is inescapable. In the next section we will see that length contraction and the twin paradox are manifestations of the reality of the four-dimensional worldtubes of the objects involved in these effects. But now we will first show how the conclusion that conventionality of simultaneity is impossible in a three-dimensional world could have been reached in 1906.

In the section 'Definition of Simultaneity' of his 1905 paper Einstein discussed the introduction of a common time at two distant points $A$ and $B$ : "We have not defined a common 'time' for $A$ and $B$, for the latter cannot be defined at all unless we establish by definition that the 'time' required by light to travel from $A$ to $B$ equals the 'time' it requires to travel from $B$ to $A$ " [37, p. 40]. This conclusion is a result of a deep analysis and shows that Einstein had re-discovered, after Poincaré, ${ }^{9}$ the unavoidable conventionality in determining the one-way velocity of light and the simultaneity of distant events. However, that conclusion raises the obvious question "How can the one-way velocity of light be a matter of definition (convention), whereas it appears to be self-evident that in reality the back and forth velocities of light are either the same or not the same?" Had Einstein pursued further his analysis he would have most probably arrived at the conclusion that the impossibility to

${ }^{9}$ In 1898 Poincaré first realized that it was a postulate that the light "velocity is the same in all directions" and that "[t]his postulate could never be verified directly by experiment" [38, p. 220]. Poincaré also arrived at the conclusion that whether two events are simultaneous is a matter of convention [38, p. 222]. 
determine the one-way velocity of light had a profound reason - reality is a four-dimensional world in which light (and anything else) does not travel at all since the whole history of a light signal is entirely realized in the (forever given) light signal's worldline.

Let us outline the way that analysis could have been performed. Undoubtedly, Einstein had realized the vicious circle when one tries to establish the simultaneity of distant events, that is, to synchronize distant clocks, with the help of light signals - to synchronize two clocks at different locations the oneway velocity of light between them should be known, but to determine the one-way velocity of light the two clocks should be synchronized beforehand. A synchronization by a slow transport of a third clock also leads to the same vicious circle. To see the vicious circle here, one should keep in mind that the third clock's velocity cannot be assumed to be zero; otherwise, it would obviously not be able to reach the clock it is trying to synchronize. No matter how small, the velocity of the third clock is different from zero, which means that the time dilation effect (no matter how small) should be taken into account. To calculate that effect the one-way velocity of the third clock should be known, but in order to determine it the two clocks, which the third clock tries to synchronize, should be synchronized in advance.

Thus, the vicious circle in determining the one-way velocity of light and therefore the simultaneity of distant events is unavoidable. Then the natural question is what message that vicious circle conveys. The deep meaning of the message becomes evident when the impossibility to determine objectively which distant events are simultaneous is analyzed in terms of what exists. Assume that reality is a three-dimensional world (everything that exists simultaneously at the present moment). As simultaneity of distant events is a matter of convention it follows that the three-dimensional world is also a matter of convention. But what exists, no matter that it is unobservable at the moment it exists, cannot be a matter of convention. Thus, the only conclusion which one can draw from here is that conventionality of simultaneity is impossible in a three-dimensional world ${ }^{10}$ (and that conclusion could have been reached before the four-dimensional formulation of special relativity was given in 1908).

Had Einstein arrived at the conclusion that simultaneity of distant events would not be established 'by definition' if reality were a three-dimensional world he would have faced quite a challenge. It is impossible to guess how much time it would have taken him to decode the message conveyed by the vicious circle and to realize that conventionality of simultaneity implies a four-dimensional world in which space and time are united into an inseparable entity. After the publication of his 1905 papers his intellectual power

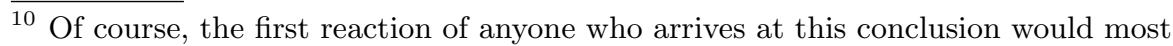
probably be to question the validity of the conventionality thesis. However, no matter how many times the analysis leading to the vicious circle would be repeated its existence would be confirmed and the conventionality thesis would follow. 
had been concentrated on making the description of gravity consistent with special relativity. Thus, it appears that the development of general relativity had prevented him from dealing with the question of the physical meaning of our freedom to define which events in the world are simultaneous. What confirms that Einstein had not pursued his analysis of the conventionality of simultaneity further is his initial negative reaction to Minkowski's fourdimensional formulation of special relativity. However, the man who was not afraid to ask fundamental questions and to seek their answers, the man who discovered the unimaginable at that time link between gravity and geometry, would have definitely been able to decode the message hidden in the vicious circle involved in any attempt to determine which events are simultaneous, if he had had the time. Most probably, Einstein would have realized that our freedom to choose our three-dimensional world (the set of simultaneous events at the moment 'now') implies that we have from where to choose. Then arriving at the idea that there is a link between conventionality of simultaneity and dimensionality of the world would not have been so unthinkable.

Once it is realized that reality is a four-dimensional world conventionality of simultaneity turns out to be trivial - as all events of spacetime are equally existent it is really our choice which events constituting a three-dimensional hypersurface (lying outside of the light cone at a given event) will be regarded as simultaneous. In such a four-dimensional world in which there are only worldlines of particles and light signals the velocity of light is just a description of light worldlines in terms of our three-dimensional language since in reality (in spacetime) light does not travel at all. If the world were threedimensional and light were really propagating, its one-way velocity could not be conventional because Nature would 'know' what is the magnitude of that velocity.

The realization that conventionality of simultaneity is another manifestation of the four-dimensionality of the world is in fact an argument against any attempt to relativize existence since such a view would preserve the threedimensionality of the world.

\subsection{The existence of accelerated observers in special relativity is impossible in a three-dimensional world}

In the early years of special and general relativity there had been some confusion about accelerated motion - for some time, due to Einstein's equivalence principle, there had been a tendency to think that it is general relativity which deals with accelerated motion. Gradually, however, the issue has been settled and in the early seventies Misner, Thorne, and Wheeler even entitled one of the sections of their Gravitation "Accelerated observers can be analyzed using special relativity" [5, p. 163]. Now the situation is completely clear general relativity describes curved spacetimes, whereas special relativity is concerned with a flat spacetime. Absolutely accelerated observers [17, Ch. 8], 
which are represented by non-geodesic worldlines, exist in both curved and flat spacetimes.

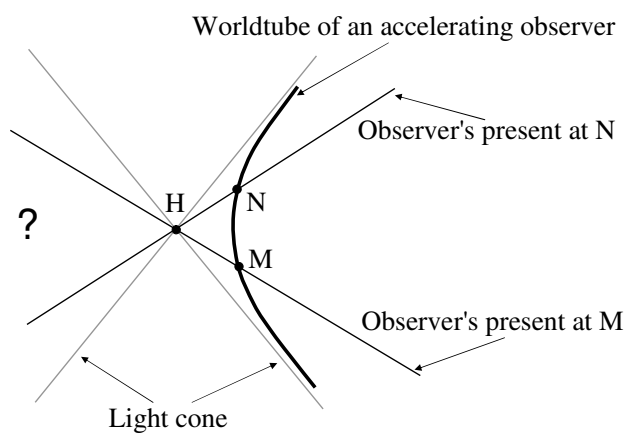

Fig. 3. At any moment of his proper time an accelerated observer has a set of simultaneous events (his present at that moment). Unlike an inertial observer's presents the presents of the accelerated observer intersect one another.

So, accelerated observers are described by special relativity, but that would not be possible if reality were a three-dimensional world. To see why this is so assume that what exists is only the present, i.e. the three-dimensional world at the moment 'now', and consider an accelerated observer whose worldtube is depicted in Fig. 3. Due to the fact that the worldtube of the accelerated observer is curved the presents that correspond to the different moments of his proper time are not parallel and intersect one another as shown in Fig. 3. Consider the accelerated observer's presents that correspond to the events $M$ and $N$. At event $M$ the spacetime region between the two presents denoted by the question mark "?" is past for the accelerated observer (including part of his present at the later event $N$ ). However, the same spacetime region lies in the future of the accelerated observer at the later event $N$ (including part of his present at the earlier event $M$ ). Obviously, this would be impossible if the world were three-dimensional. Thus, the existence of accelerated observers in special relativity is another manifestation of the four-dimensionality of the world and is therefore an argument against the view that existence should be relativized because this view regards the world as three-dimensional.

Almost certainly a physicist would object to the above argument by pointing out that there are constrains on the size of an accelerating frame in relativity [5, p. 168]. These constrains result from the fact that a coordinate system associated with the accelerated observer cannot be extended to the left in Fig. 3 beyond event $H$. The first objection against using a global coordinate system in the case of an accelerated observer was mentioned above - coordinate time makes no sense beyond event $H$ because what is past time at event $M$ (in the region "?") is future time at the later event $N$. A second objection is the fact that the coordinates of events located in the spacetime 
region between the past and future light cones, which contains the region "?", cannot be determined by sending and receiving light signals [5, p. 168]. The event $H$ (in fact, a two-dimensional surface) acts as a horizon for the accelerated observer - no signals can be received from and sent to the spacetime region lying to the left of that event.

All this is correct, but the above two objections are concerned only with the description of the events of spacetime and have nothing to do with the question of whether or not they exist. That the impossibility for an accelerated observer to communicate with events located to the left of event $H$ (between the past and future light cone at $H$ ) has no effect on the existence of these events is best demonstrated by the fact that all comoving inertial observers at the different events of the worldtube of the accelerated observer can communicate with those events. And if they exist for the comoving inertial observers they should exist for the accelerated observer as well.

It should be noted here that the relativistic division of events into past, present, and future (Fig. 1), which defines the notion of a light cone, reveals only the causal structure of spacetime and has no relation to the existence of the spacetime events ${ }^{11}$. As we have seen above if one assumes that the events located outside of the light cone at an event exist it is easily shown that all spacetime events exist.

\section{Physical objects are four-dimensional worldtubes}

In this section we will be concerned with the dimensionality of the objects involved in the relativistic effects. This issue was dealt with in more detail in [17, Ch. 5]. Here I will discuss the relativistic length contraction by addressing Ohanian's objections [33] to the account given in [17] and the twin paradox by examining another version of it that rules out the acceleration as a cause for the time difference observed by the twins when they meet.

\subsection{Length contraction would be impossible if the contracting meter stick were a three-dimensional object}

It is unfortunate that the physics papers and books on relativity do not discuss the issue of the dimensionality of the objects subjected to relativistic effects. As discussed in the Introduction the most probable explanation might be that that issue is regarded by physicists as philosophical. However, I wonder how many physicists, if directly asked, would subscribe to the view that the dimensionality of physical objects (and the world) is a philosophical question.

If the dimensionality issue is explicitly addressed, not only the description, but a full explanation of these effects can be achieved. Take as an example length contraction. Consider a meter stick at rest with respect to an observer

$\overline{11}$ One can think of a four-dimensional world with no causal structure. 


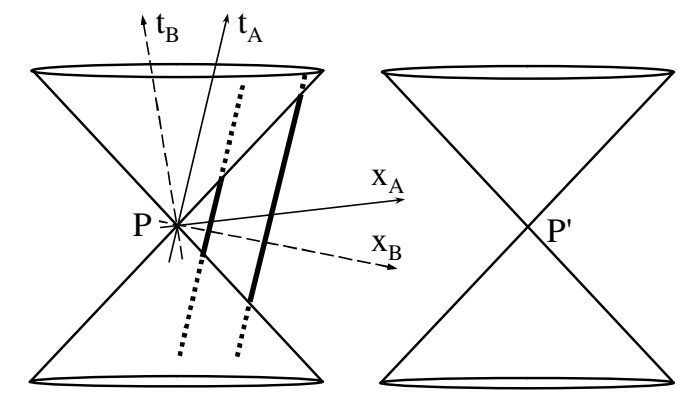

Fig. 4. A meter stick, whose end points' worldlines are represented by the thick lines, is at rest with respect to observer $A$. The instantaneous space of observer $B$ at event $P$ intersects the worldtube of the meter stick in a three-dimensional crosssection, whose length is shorter than the cross-section 'cut off' by the instantaneous space of observer $A$. If one assumes that only the part of the meter stick's worldtube which lies in the spacetime region outside of the light cone at $P$ is real, then by the same argument the worldtube's parts that are in the past and future light cone at $P$ are also real since they lie outside of a second light cone at $P^{\prime}$.

$A$ (Fig. 4). A second observer $B$ moving relative to $A$ finds that the length of the meter stick is relativistically contracted. The natural question is "What is the physical meaning of the relativistic length contraction?" Physicists usually avoid addressing it by pointing out that what a physicist should be concerned with is its derivation through the Lorentz transformations. Although many physicists believe that the ultimate goal of physics is understanding of the real world (and do not share this purely descriptive role of physics), it is worth noting here that the very derivation of this effect from the Lorentz transformations involves an overlooked subtlety ${ }^{12}$ [17, pp. 94-97] which clearly raises the question of the physical meaning of length contraction. This subtlety

${ }^{12}$ The subtlety involves an unusual use of the Lorentz transformations in the derivation of length contraction. Instead of performing what appears to be the correct transformation $A \Rightarrow B$ (which expresses the unknown in frame $B$ coordinates of the end points of the meter stick as a function of their known in frame $A$ coordinates) this effect is derived through the transformation $B \Rightarrow A$, i.e. by expressing the known coordinates of the meter stick's end points through their unknown coordinates. The requirement that the end points of the meter stick should be measured simultaneously in $B$ cannot be used as a justification for the transformation $B \Rightarrow A$ since the transformation $A \Rightarrow B$ also ensures the simultaneity of the events of the measurement in $B$ [17, p. 95]. (For comparison consider a process that takes place at a point in $A$ and has a duration $t_{A}$ in $A$. In order to determine its relativistically dilated duration $t_{B}$ in $B$, observer $B$ performs the 'correct' Lorentz transformation $A \Rightarrow B$, not $B \Rightarrow A$.) The Lorentz transformation $B \Rightarrow A$ gives the correct length contraction because it is only this transformation that relates the end points of two three-dimensional cross-section of the worldtube of the meter stick; the transformation $A \Rightarrow B$ does not link pairs of events that constitute cross-sections of the meter stick's worldtube. This fact 
implies that the meter stick is not a three-dimensional object, but a fourdimensional worldtube - the three-dimensional meter stick equally existing at all moments of its history. As a result, while measuring the same meter stick the two observers measure two different three-dimensional objects, which are different three-dimensional cross-sections of the meter stick's worldtube. This is shown in Fig. 4. The instantaneous spaces of $A$ and $B$ 'cut off' different three-dimensional cross-sections from the worldtube of the meter stick and $A$ 's cross-section, representing the proper length of the meter stick, turns out to be the longest of all possible cross-sections. Thus, the realization that what we perceive as a three-dimensional meter stick is in fact a four-dimensional worldtube provides a $100 \%$ explanation ${ }^{13}$ of the relativistic length contraction - the instantaneous space of $B$ intersects the meter stick's worldtube in a three-dimensional cross-section which is different from and shorter than the cross-section of $A$.

A possible objection to this 'a $100 \%$ explanation' of the relativistic length contraction is that one should not take the spacetime time diagram shown in Fig. 4 too seriously. First, it is the analysis of the derivation of this effect which demonstrates that what is depicted in Fig. 4 adequately represents the dimensionality of the meter stick. Second, the conclusion that $A$ and $B$ measure two different three-dimensional objects follows directly from relativity of simultaneity when it is taken into account that the meter stick as an extended body is defined as the set of its 'parts' which exist simultaneously at a given moment. Since the observers $A$ and $B$ are in relative motion they have different sets of simultaneous events and therefore different three-dimensional meter sticks. It is evident from here that the worldtube of the meter stick must be real in order that $A$ and $B$ consider different three-dimensional crosssections of it as their three-dimensional meter sticks. Otherwise, if the meter stick were what everyone is tempted to assume as self-evident - a single threedimensional object - (just $A$ 's meter stick), no length contraction would be possible because that single three-dimensional object would constitute a single set of simultaneous events, which would be common to all observers in relative motion in contradiction with special relativity.

Here, one could again raise the objection that the conclusion of the reality of the meter stick's worldtube is based on relativity of simultaneity, whereas one is free to choose any hypersurface in the spacetime region outside of the light cone at event $P$ (Fig. 4). However, like in the general case of the

implies that the worldtube of the meter stick is a real four-dimensional object; if this were not the case than the transformation $A \Rightarrow B$ would be used which would lead to internal inconsistencies in special relativity and ultimately to contradictions with experiments that confirmed the relativistic length contraction (e.g. the muon experiment, which tested both time dilation and length contraction [39]).

${ }^{13}$ I call it 'a $100 \%$ explanation' since it is not based on any other explicit or implicit assumptions. If the worldtube of the meter stick is real, that is all - it completely explains the physical meaning of length contraction. The 'if' is convincingly removed as we will see below. 
dimensionality of the world discussed in Section 2 this objection is, in fact, directed against the view that the meter stick is a three-dimensional object since a three-dimensional object is defined in terms of simultaneity ${ }^{14}$. The freedom to choose any hypersurface which lies outside of the light cone, i.e. the freedom to choose any cross-section of the meter stick's worldtube outside of the light cone, means that the part of the worldtube (depicted with thick lines) located outside of the light cone must be real in order to have from where to choose. The rest of the meter stick's worldtube (represented by the dashed lines) which lies in the past and the future light cone at $P$ must also be real since it is outside of another light cone associated with event $P^{\prime}$ (Fig. 4).

Thus, the conclusion that the worldtube of the meter stick is a real fourdimensional object is inescapable. To realize this even better let us ask the question "What is the dimensionality of the meter stick itself (not what we see or measure $\left.{ }^{15}\right)$ ?" Obviously, there are only three possibilities for the dimensionality of the meter stick: (i) a three-dimensional object, (ii) part of the worldtube of the meter stick which lies outside of the light cone at event $P$ (Fig. 4), and (iii) the entire worldtube of the meter stick. As we have seen above (i) contradicts relativity, whereas (ii) leads to (iii):

- If the meter stick is a three-dimensional object (at rest with respect to observer $A$ ) relativity of simultaneity is impossible since it follows from relativity of simultaneity that $A$ and $B$ measure two different threedimensional objects, which is only possible if the meter stick's worldtube exists.

- If the meter stick is the part of its worldtube which lies outside of the light cone at $P$ then it follows that the entire worldtube of the meter stick is real since the parts of the worldtube that lie in the past and future light

${ }^{14}$ The fact that no intrinsic (therefore frame-independent) feature of spacetime can be associated with the notion 'simultaneity in spacetime', i.e. with the notion 'distant present events', demonstrates that the concept of a three-dimensional object (which is defined in terms of simultaneity) has no place in relativity. This conclusion appears to be in such an obvious contradiction with our common sense view on physical objects that the status of three-dimensional objects in relativity has been avoided so far.

${ }^{15}$ What the observers $A$ and $B$ see when they meet momentarily at $P$ (Fig. 4 ) is the same three-dimensional cross-section of the meter stick's worldtube of the same length, but it does not constitute a three-dimensional object since that image is the intersection of the past light cone with the worldtube of the meter stick, whereas a three-dimensional object is the collection of all 'parts' of the object that exist simultaneously at a given moment of an observer's time [32]. A thought experiment [17, p. 137] involving instantaneous measurements demonstrates that two observers in relative motion do measure two different three-dimensional objects. Thus, not only the theoretical prediction of relativistic length contraction would not be possible but any experiments that test this effect would be also impossible if the meter stick's worldtube were not real. 
cone at $P$ (and therefore are regarded as non-existent for an observer at $P$ ) lie outside of another light cone at $P^{\prime}$ and therefore are regarded as existent for an observer at $P^{\prime}$ (Fig. 4).

As we have seen not only does the assumption that the worldtube of the meter stick is real provide a natural and complete explanation of the relativistic length contraction, but also that explanation is the only one that is consistent with relativity itself. A striking feature of this explanation is that it is not dynamical since it turns out to be a manifestation of the fourdimensionality of the world and in particular a manifestation of the fact that the meter stick itself is a four-dimensional worldtube, not a three-dimensional object. However, the assumption of the reality of the worldtube of the meter stick is so counter-intuitive that after the original Lorentz-FitzGerald explanation of the length contraction effect, which involved a deformation of the meter stick caused by forces acting on its atoms, there have always been attempts to provide a dynamical explanation of the relativistic length contraction which should account for the deformation of the meter stick (see for example [33], [40], [41]). Such an explanation, however, is in an insurmountable contradiction with relativity of simultaneity since it presupposes that two observers in relative motion have a common set of simultaneous events - the observers measure the length of the same three-dimensional object, i.e. the same set of simultaneous events, which constitute the meter stick.

Another argument which demonstrates the failure of any dynamical explanation of the relativistic length contraction is the fact that such an explanation cannot account for the contraction of space itself where there are no atoms and forces acting between them [17, pp. 135-136]. However, Ohanian [33] disagrees with this argument ${ }^{16}$ : "If the length contraction represents anything physical, it must be a contraction of physical bodies, not a 'contraction

${ }^{16}$ Ohanian also writes: "We could, in principle, do physics in the manner of Lorentz, in one single coordinate system (what Lorentz called the ether frame)" [33]. This could not be done since relativity of simultaneity and reciprocity of length contraction and time dilation require two inertial frames. Otherwise one could not talk about proper length (in the rest frame) and contracted length (in a frame moving relative to the rest frame) and about proper time and dilated time (which again require two frames). Lorentz himself admitted the failure of his attempt to use just one frame (the ether frame) in which the coordinates $x, y, z$, and $t$ were the true coordinates, whereas the quantities $x^{\prime}, y^{\prime}, z^{\prime}$ and $t^{\prime}$ were nothing more than mathematical quantities [42]:

The chief cause of my failure was my clinging to the idea that the variable $t$ only can be considered as the true time and that my local time $t^{\prime}$ must be regarded as no more than an auxiliary mathematical quantity. In Einstein's theory, on the contrary, $t^{\prime}$ plays the same part as $t$; if we want to describe phenomena in terms of $x^{\prime}, y^{\prime}, z^{\prime}, t^{\prime}$ we must work with these variables exactly as we could do with $x, y, z, t$. 
of space". I do not think this objection can be defended in the framework of relativity. Nowhere in special relativity there is any requirement that the Lorentz transformations must be applied to physical bodies only. The distance between two points of space contracts ${ }^{17}$ relativistically as well. Otherwise, if only physical bodies contracted, one would arrive at the following paradoxical result (which can be found in some explanations of the geometry on a rotating disk). Consider the distance $L_{M N}$ between two points $M$ and $N$ in space, say the distance between two objects at rest with respect to an observer $A$. Using a meter stick $A$ determines that $L_{M N}=10 \mathrm{~m}$. A second observer $B$ moving relative to $A$ applies the Lorentz transformations only to the physical meter stick and concludes that due to its relativistic contraction more meter sticks will fit between the points $M$ and $N$ and as a result the distance $L_{M N}$ will be greater that $10 \mathrm{~m}$, i.e. it will be relativistically dilated for $B$, not contracted. One can arrive at more paradoxical results if the assumption that only physical bodies contract is carefully analyzed.

\subsection{The twin paradox would be impossible if the twins were three-dimensional bodies}

The best way to prove the statement in the section title is to accept for a moment the common view on the dimensionality of physical bodies and assume that each of the twins $A$ and $B$, represented by their worldtubes in Fig. 5 , is a three-dimensional body that exists only at the moment 'now' of the proper time of the twin. On this view the twins' worldtubes are not real fourdimensional objects; these are nothing more than graphical representations of the twins' histories, which do not have counterparts in the external world. On the three-dimensionalist view time flows objectively which means that any discrepancy in the readings of the twins' clocks results from a different 'rate' of the twins' times. When $A$ and $B$ meet at event $M$ they will determine that twin $B$ is, say, five years younger than his brother. As the twins exist as threedimensional bodies only at $M$ the only explanation of the five-year difference in their times is an objective slowing down of $B$ 's time. The spacetime explanation that $B$ 's worldtube is shorter ${ }^{18}$ than $A$ 's worldtube cannot be used since we started with the assumption that the twins exist as three-dimensional bodies, not worldtubes.

The only cause for the slowing down of twin $B$ 's time could be the acceleration to which he is subjected four times during his journey - acceleration at $D$ when he departs, deceleration at $T$ when he stops to turn back, acceleration at $T$ on his way back to twin $A$, and final deceleration when he stops to meet

${ }^{17}$ And that distance can be measured by light signals, not only by physical bodies (meter sticks).

${ }^{18}$ In Fig. 5 twin $B$ 's worldtube is longer but this is due to the fact that a situation in the pseudo-Euclidean Minkowski spacetime is represented on the Euclidean surface of the paper. 


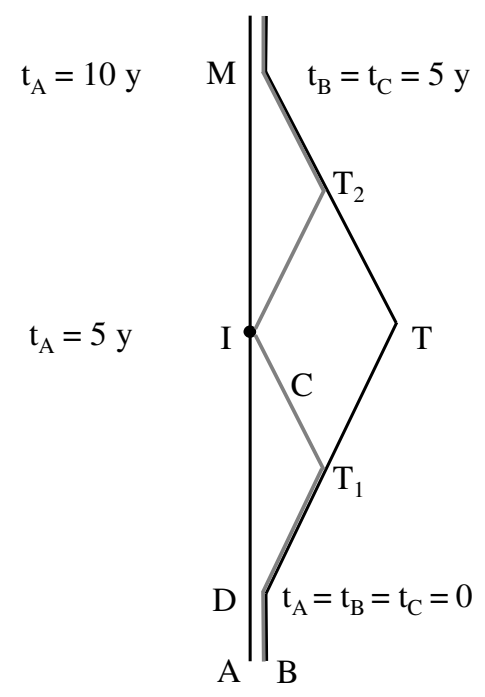

Fig. 5. Twins $A$ and $B$, whose worldtubes are represented by the black lines, separate at event $D$ and meet at event $M$. A third twin $C$, whose worldtube is depicted by the grey line, also departs at $D$ and initially moves with $B$ but at event $T_{1}$ turns back and after reaching $A$ at event $I$ departs again, intercepts $B$ at event $T_{2}$, turns back and together with $B$ meets $A$ at event $M$.

his brother at $M$. However, it has been determined that acceleration does not cause the time difference in $A$ 's and $B$ 's clocks' readings at $M$ by both (i) the experiments (see $[14$, p. 83]) which tested the so-called 'clock hypothesis' [5, p. 164], [11, p. 52], [20, p. 33] according to which the rate of an ideal clock is not affected by its acceleration, and (ii) the three-clock (or three-twin) version of the twin paradox (see, for instance, [43]). A third argument also involves a third twin $C$ (Fig. 5) who, however, is not inertial since he accelerates eight times - at events $D$ and $M$ twin $C$ has the same regime of acceleration as twin $B$ at these events, at events $T_{1}$ and $T_{2}$ his acceleration regime is as the acceleration of $B$ at $T$, and at event $I$ twin $C$ 's acceleration is a mirror image of $B$ 's acceleration at $T$. Despite that $C$ experiences more instances of acceleration, there will be again a five-year difference in $A$ 's and $C$ 's times when he arrives at $M$. This shows that the acceleration does not cause any slowing down of time. Therefore, in the case of the standard version of the twin paradox $B$ 's acceleration cannot be the cause for the slowing down of his time, which means that his time flows at the same 'rate' as twin $A$ 's time. The conclusion is that at event $M$ the twins will be of the same age. Thus, the twin paradox would not be possible if the twins were three-dimensional bodies which existed only at the present moments of the twins.

The same conclusion that $A$ and $B$ will be of equal age at $M$, if they are three-dimensional bodies existing only at $M$, also follows from the fact that 
$A$ and $B$ measure proper times. When the twins meet at $M$ they compare their proper times [17, pp. 144-145], but the proper time does not change relativistically. Therefore, if the twins existed only at the event $M$ as threedimensional bodies the objective flow of $A$ 's and $B$ 's times (possible only in a three-dimensional world) would be the same which means that the twins would be of the same age at $M$. Thus, the twin paradox is impossible as a theoretical and an experimental result if it is assumed that the twins' worldtubes are not real and the twins are the observable three-dimensional bodies that exist only at the constantly changing present moments of the twins' times. This results also rules out any attempt to relativize the existence of physical bodies since such a relativization of existence preserves the three-dimensionality of the world and the physical bodies.

The only non-contradictory explanation ${ }^{19}$ of the twin paradox can be given by acknowledging the existence of the twins' worldtubes. Then this effect is simply the triangle inequality in the pseudo-Euclidean Minkowski spacetime. Twin $B$ is younger than $A$ at $M$ since his worldtube between the events $D$ and $M$ is shorter than $A$ 's worldtube between the same events. This is also a $100 \%$ explanation. It makes it evident why the acceleration is not the cause of the time difference between $A$ 's and $B$ 's clock readings at $M$ - in spacetime the acceleration of a body is represented by a curvature of the body's worldtube, but a curvature does not change the length of a worldtube (i.e. a body's proper time). As seen in Fig. 5 the lengths of the worldtubes of twins $B$ and $C$ between events $D$ and $M$ are the same (the segment $T_{1} I$ of $C$ 's worldtube is equal $T T_{2}$ of $B$ 's worldtube and $I T_{2}$ of $C$ 's worldtube is equal to $T_{1} T$ of $B$ 's worldtube). That is why $B$ and $C$ are of the same age at $M$.

\section{Conclusions}

The main aim of the paper is to demonstrate that the kinematical relativistic effects are manifestations of the four-dimensionality of the world. As such these effects would be impossible if the world were three-dimensional. This was shown in the cases of relativity of simultaneity, conventionality of simultaneity, accelerated observers in special relativity, length contraction, and the twin paradox. Therefore, the concept of a three-dimensional world contradicts not only special relativity (as a theory) but more importantly the experimental evidence which supports it.

I would like specifically to stress that no appeal to quantum mechanics or any future theories (e.g. quantum gravity) can change the fact of the contradiction of the three-dimensionalist view with the experiments which confirmed the kinematical consequences of special relativity.

19 This relativistic effect was initially described in three-dimensional language. But when the question of its physical meaning is raised it becomes evident that the twin paradox is a manifestation of the reality of the twins' worldtubes and the four-dimensionality of the world. 
We are approaching the one hundredth anniversary of Minkowski's talk on spacetime, but the essence of the new world view he advocated has turned out to be difficult to accept. That is why let me conclude with a quote from Eddington [44]:

However successful the theory of a four dimensional world may be, it is difficult to ignore a voice inside us which whispers: "At the back of your mind, you know that a fourth dimension is all nonsense." I fancy that that voice must often have had a busy time in the past history of physics. What nonsense to say that this solid table on which I am writing is a collection of electrons moving with prodigious speeds in empty spaces, which relatively to electronic dimensions are as wide as the spaces between the planets in the solar system! What nonsense to say that the thin air is trying to crush my body with a load of $14 \mathrm{lbs}$. to the square inch! What nonsense that the star cluster which I see through the telescope obviously there now, is a glimpse into a past age 50,000 years ago! Let us not be beguiled by this voice. It is discredited.

\section{Acknowledgements}

Most of the ideas in this paper were presented at a two-day workshop of the Canadian Strategic Knowledge Cluster TaU, Time and Universe (sponsored by the Social Sciences and Humanities Research Council of Canada) and its international associates, which was held at Concordia University, Montreal on June 12-13, 2006. I would like to thank the colleagues who attended the workshop and took part in the discussion after my talk.

\section{References}

1. H. Minkowski: Space and Time. In: H.A. Lorentz, A. Einstein, H. Minkowski, and H. Weyl: The Principle of Relativity: A Collection of Original Memoirs on the Special and General Theory of Relativity (Dover, New York 1952) pp 75-91

2. J.L. Synge: Relativity: The Special Theory, 2nd edn (North-Holland, Amsterdam 1965)

3. J.L. Synge: Relativity: The General Theory (North-Holland, Amsterdam 1960)

4. E.F. Taylor, J.A. Wheeler: Spacetime Physics, 2nd edn (W. H. Freeman, New York 1992)

5. C.W. Misner, K.S. Thorne, and J.A. Wheeler: Gravitation (W. H. Freeman, New York 1973)

6. E.F. Taylor, J.A. Wheeler: Exploring Black Holes: Introduction to General Relativity (Addison Wesley Longman, New York 2000)

7. B. Schutz: Gravity from the Ground Up: An Introductory Guide to Gravity and General Relativity (Cambridge University Press, Cambridge 2003) 
8. J.B. Hartle: Gravity: An Introduction to Einstein's General Relativity (Addison Wesley, San Francisco 2003)

9. S.M. Carroll: Spacetime and Geometry: An Introduction to General Relativity (Addison Wesley, San Francisco 2004)

10. W. Rindler: Relativity: Special, General and Cosmological (Oxford University Press, Oxford New York 2001) p 91

11. G.L. Naber: The Geometry of Minkowski Spacetime: An Introduction to the Mathematics of the Special Theory of Relativity (Springer, Berlin Heidelberg New York 1992)

12. A. Das: The Special Theory of Relativity: A Mathematical Exposition (Springer, Berlin Heidelberg New York 1993)

13. E.G. Peter Rowe: Geometrical Physics in Minkowski Spacetime (Springer, Berlin Heidelberg New York 2001) p 3

14. R. A. Mould: Basic Relativity (Springer, Berlin Heidelberg New York 1994)

15. D.-E. Liebscher: The Geometry of Time (Wiley-VCH, Weinheim 2005) p 5

16. W.S.C. Williams: Introducing Special Relativity (Taylor and Francis, London 2002)

17. V. Petkov: Relativity and the Nature of Spacetime (Springer, Berlin Heidelberg New York 2005)

18. N. Straumann: General Relativity With Applications to Astrophysics (Springer, Berlin Heidelberg New York 2004) p 46

19. M.P. Hobson, G. Efstathiou, A.N. Lasenby: General Relativity: An Introduction for Physicists (Cambridge University Press, Cambridge 2006) pp 125, 357

20. R. d'Inverno: Introducing Einstein's Relativity (Clarendon Press, Oxford 1992) p 17

21. J.J. Callahan: The Geometry of Spacetime: An Introduction to Special and General Relativity (Springer, Berlin Heidelberg New York 2000) p 10

22. G. Barton: Introduction to the Relativity Principle (Wiley, New York 1999) p 10

23. T.-P. Cheng: Relativity, Gravitation and Cosmology (Oxford University Press, Oxford New York 2005) p 5

24. C. W. Rietdijk: Philosophy of Science 33341 (1966)

25. H. Putnam: Journal of Philosophy 64, 240 (1967)

26. N. Maxwell: Philosophy of Science 52, 23 (1985)

27. A. Einstein: Relativity: The Special and General Theory (Crown Publishers, New York 1961) p 150

28. H. Weyl: Philosophy of Mathematics and Natural Science (Princeton University Press, Princeton 1949) p 116

29. R. Weingard: British Journal for the Philosophy of Science 23, 119 (1972)

30. H. Stein: Journal of Philosophy 65, 5 (1968)

31. H. Stein: Philosophy of Science 58, 147 (1991)

32. V. Petkov: Is There an Alternative to the Block Universe View? In: The Ontology of Spacetime, ed by D. Dieks (Elsevier, Amsterdam 2006) pp 207-228

33. H.C. Ohanian: The Real World and Spacetime. In: this volume.

34. R.M. Wald: American Journal of Physics 74, 471 (2006)

35. V. Petkov: British Journal for the Philosophy of Science 40, 69 (1989)

36. J. Winnie: Philosophy of Science 37, 81 (1970); Philosophy of Science 37, 223 (1970) 
37. A. Einstein: On the electrodynamics of moving bodies. In: H.A. Lorentz, A. Einstein, H. Minkowski, and H. Weyl: The Principle of Relativity: A Collection of Original Memoirs on the Special and General Theory of Relativity (Dover, New York 1952) pp 37-65

38. H. Poincaré: The Value of Science (The Modern Library, New York 2001) pp $210-222$

39. G.F.R. Ellis and R.M. Williams: Flat and Curved Space Times (Oxford University Press, Oxford 1988) p 104

40. J.S. Bell: How to teach special relativity. In: J. S. Bell: Speakable and Unspeakable in Quantum Mechanics (Cambridge University Press, Cambridge 1987) pp $67-80$

41. H.R. Brown: Physical Relativity: Spacetime Structure from a Dynamical Perspective (Clarendon Press, Oxford 2005)

42. H.A. Lorentz: The Theory of Electrons and Its Applications to the Phenomena of Light and Radiant Heat, 2nd ed. (Dover, Mineola New York 2003) p 321

43. P. Kroes: Philosophy of Science 50, 159 (1983)

44. A.S. Eddington: Space, Time and Gravitation: An Outline of the General Relativity Theory (Cambridge University Press, Cambridge 1920) p 56 